\title{
Über die retikulären Zellen im Gehirn des japanischen Dornhaies (Acanthias mitsukurii Jordan et Fowler).
}

\author{
Von \\ Tamesuke Saito. \\ Aus dem Anatomischen Institut der Keio Universität, Tokyo.
}

Mit 9 Textfiguren.

\section{Einleitung.}

Untersuchungen über die retikulären Zellen im Gehirn der Plagiostomen, besonders des Dornhaies, wurden bisher von sehr wenigen Autoren unternommen. Mitteilungen brachten nur Kappers('06) bei Selachiern über die retikulären Fasern, Sterzi ('09) bei Acanthias und van Hoevell ('11) bei Rjaa clavata über die betreffenden Zellen, Kappers('20 u. '21) bei Plagiostomen über die beiden und Kuhlenbeck('27) bei Selachiern über ihre Bedeutung im Rahmen des Bauplans.

In der vorliegenden Untersuchung habe ich besonders meine Aufmerksamkeit auf die Einteilung, Grösse, Anordnung, Lokalisation und den Faserverlauf der retikulären Zellen im Zentralnervensystem des japanischen Dornhaies gelenkt, worüber ich im folgenden berichten werde.

\section{Material und Methode.}

Als Material habe ich erwachsene Exemplare vom japanischen Dornhai (Acanthias), Squalus mitsukurii Jordan et Fowler, untersucht.

Als Fixierungsmittel wurde Müllersche Lösung angewendet.

Nach der Zelloidineinbettung wurden sagittale und frontale Serienschnitte von $50 \mu$ Dicke angefertigt. Die Färbung geschal nach der Palschen Markscheidenmethode.

Ferner habe ich nach Paraffineinbettung des mit Alkohol fixierten Materials $12 \mu$ dicke sagittale und frontale Serienschnitte angefertigt, die 
mit $0.1 \%$ iger Toluidinblaulösung (Ganglienzellenfärbung) gefärbt wurden. Endlich wurde noch Material nach Formalinfixierung und Boraxkarminstückfärbung im Zelloidin eingebettet und in $50 \mu$ dicke frontale und sagittale Serienschnitte geschnitten.

Ich behandelte die Schnitte mit einer stärkeren (0.5-0.75\%) Kalipermanganatlösung 5-10 Minuten lang oder noch länger und differenzierte 1-2 Stunden lang; dann sind die Markscheiden dunkelblau und die retikulären Zellen schwarzbraun gefärbt. Die letzteren lassen sich so scharf von sonstigen Ganglienzellen und Nervenfasern unterscheiden.

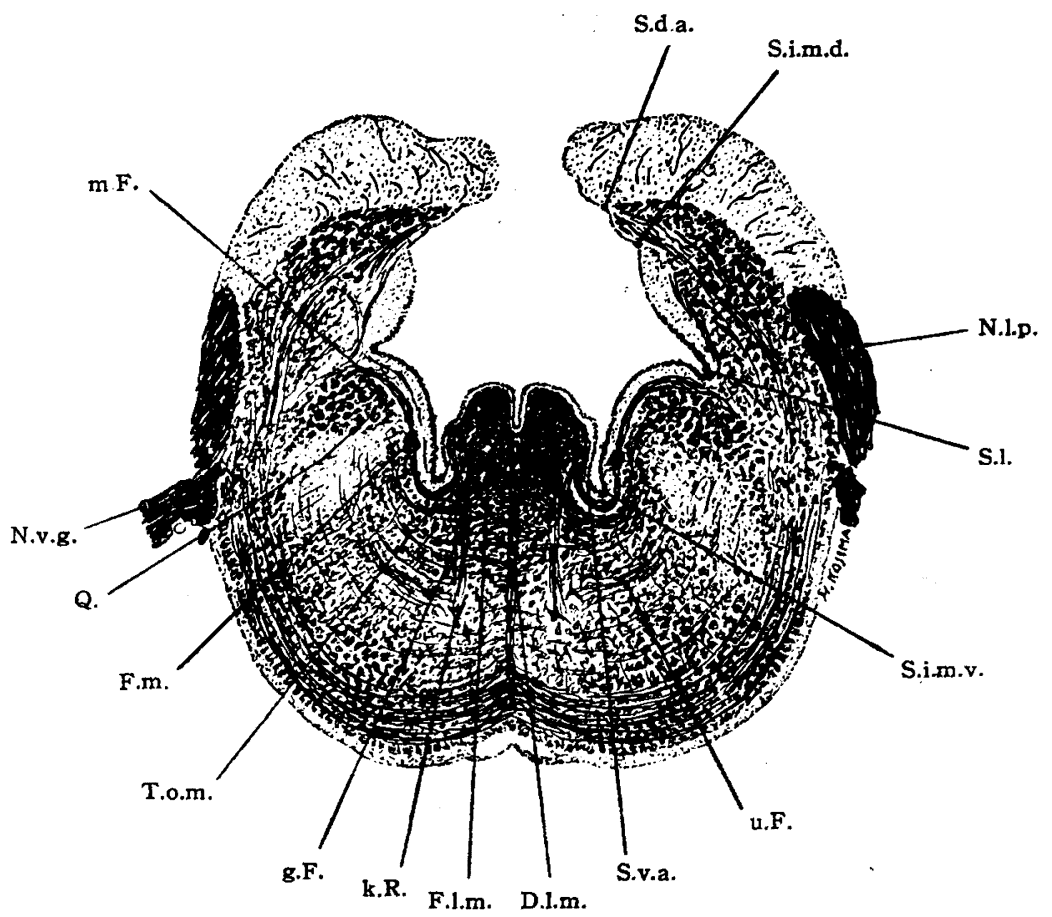

Fig. 1.

Kaudale Rhombencephalonuntergruppe der retikulären Zellen, Tractus reticularis und Tractus octavo-motorius an der Eminentia posterior. D.l.m. Decussatio longitudinalis mediana. F.l.m. Fasciculus longitudinalis mediulis, F.m. Fasciculus medianus, g.F. gekreuzte Fasern der retikulären Zellen, k.R. kaudale Rhombencephalonuntergruppe der retikulären Zellen, m.F. motorische Fasern des Nervus facialis, N.l.p. Nervus lateralis posterior, N.v.g. Nervus vagus und glossopharyngeus, Q. Quintus, S.d.a. Sulcus dorsalis accessorius, S.i.m.d. Sulcus intermedius dorsalis, S.i.m.v. Sulcus intermedius ventralis, S.l. Sulcus limitans, S.v.a. Sulcus ventralis accessorius, T.o.m. Tractus octavomotorius, u.F. ungekreuzte Fasern des Tractus reticularis. 


\section{Eigene Befunde.}

1. Vorhandensein der Zellen.

Die retikulären Zellen liegen immer im grössten Teil des Rhombencephalon d.h. in der eigentlichen Medulla oblongata ${ }^{1)}$, im rostralen Abschnitt des Rhombencephalon ${ }^{2)}$ sowie im Mesencephalon ${ }^{3)}$, und nicht im Übergangsgebiet des Rhombencephalon in das Rückeṇmark ${ }^{4)}$, noch im Diencephalon ${ }^{5)}$ und Telencephalon ${ }^{\text {() }}$.

Die Zellen sind fast durch die ganzen Länge der eigentlichen $\mathrm{Me}-$ dulla oblongata vorhanden. Während die Zellen in der Gegend der hinteren und vorderen viszeralen Zellsäule d.h. in der Eminentia anterior ${ }^{\text {t) }}$ (Fig. 2 u. 3) und posterior ${ }^{8}$ (Fig. 1) sich reichlich ansammeln, wie die sonstigen Ganglienzellen, fehlen sie in der Zwischengegend der beiden Eminentiae gänzlich oder sind nur sehr spärlich vorhanden. Kaudal von der Eminentia posterior konnte ich sie nicht konstatieren. Die eigentliche Medulla oblongata, besonders die Eminentia anterior enthalten die Zellen am reichlichsten unter allen Gehirnteilen.

Im rostralen Abschnitt des Rhombencephalon, besonders in der Isthmusgegend (Fig. 4) befinden sich die Zellen am wenigsten zerstreut.

Auch im Mesencephalon (Fig. 8.) befinden sich wenig Zellen, wobei sie zwischen den Oculomotoriuswurzelfasern, besonders im rostralen Teil, und der Commissura transversa nur wenig gruppiert vorhanden sind.

\section{Einteilung.}

Die retikulären Zellen werden in folgende drei Gruppen eingeteilt : die Mesencephalongruppe (Fig. 8), die Isthmusgruppe (Fig. 4) und die Rhombencephalongruppe (Fig. 1-3). Die letztere teilt sich wieder in eine rostrale (Fig. 2 u. 3) und eine kaudale Untergruppe (Fig. 1). Die rostrale Untergruppe liegt in der Eminentia anterior, die kaudale Untergruppe befindet sich in der Eminentia posterior.

1), 2), 3), 4), 5) u. 6). Die Grenze des Rhombencephalon, Mesencephalon, Diencephalon und Telencephalon, besonders zwischen den beiden letzteren, stimmt mit der beim Gehirn des japanischen Flussneunauges überein (Über das Gehirn des japanischen Flussneunauges, Entosphenus japonicus Marten s, Folia Anatomica Japonica, Band VIII, Heft 3-4, '30). Nur wird das Übergangsgebiet des Rhombencephalon in das Rückenmark rostral mit dem Calamus, kaudal mit der letzten d.h. vierten Spinooccipitalnervenwurzel begrenzt; durch den ersten Spinooccipitalnerven wird dieses Gebiet in eine Pars anterior und eine Pars posterior eingeteilt.

7) u. 8). Eminentia anterior und posterior verhalten sich ganz gleich wie beim japanischen Flussneunauge. 


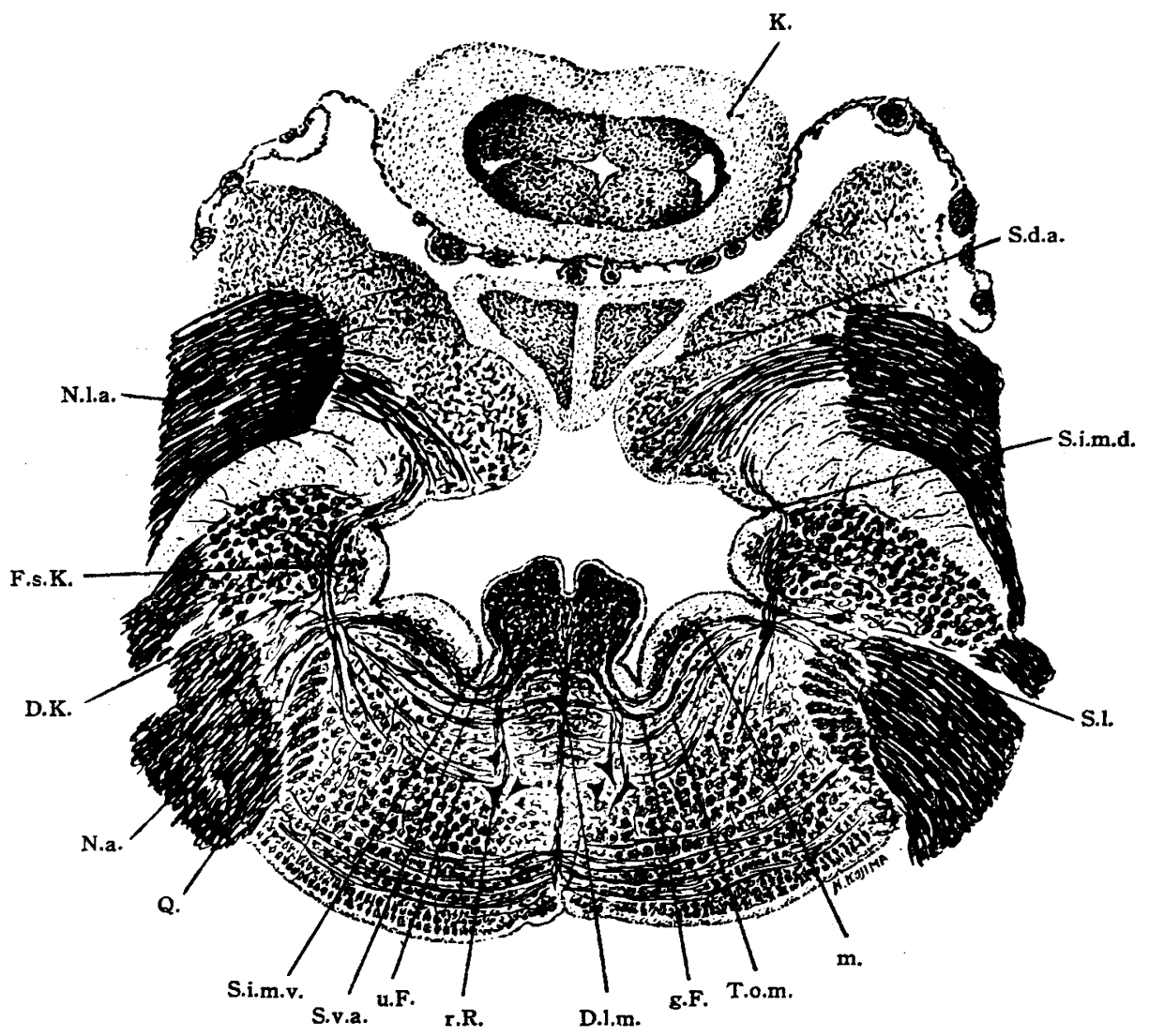

Fig. 2.

Rostrale Rhombencephalonuntergruppe der retikulären Zellen, Deitersscher Kern und Tractus reticularis an der Eminentia anterior. D.K. Deiters scher Kern, D.l.m. Decussatio longitudinalis mediana, F.s.K. Facialis sensibler Kern, g.F. gekreuzte Fasern des Tractus reticularis, $K$. K'leinhirn, m. Facialis motorische Fasern, N.a. Nervus acusticus, N.l.a. Nervus lateralis anterior, Q. Quintus, r.R. rostrale Rhombencephalonuntergruppe der retikulären Zellen, S.d.a. Sulcus dorsalis accessorius, S.i.m.d. Sulcus intermedius dorsalis, S.i.m.v. Sulcus intermedius ventralis, S.l. Sulcus limitans, S.v.a. Sulcus ventralis accessorius, T.o.m. Tractus octavo-motorius, u.F. ungekreuzte Fasern des Tractus reticularis.

Es ist nicht so schwer, die einzelnen Gruppen voneinander zu unterscheiden, weil zwischen der rostralen und kaudalen Rhombencephalonuntergruppe, zwischen den Rhombencephalongruppen und der Isthmusgruppe und zwischen dieser letzteren und der Mesencephalongruppe je ziemlich weite $Z$ wischenräume zu sehen sind.

In naher Beziehung zur rostralen Rhombencephalonuntergruppe steht der unmittelbar dorsal des Sulcus limitans gelegene Deiterssche 


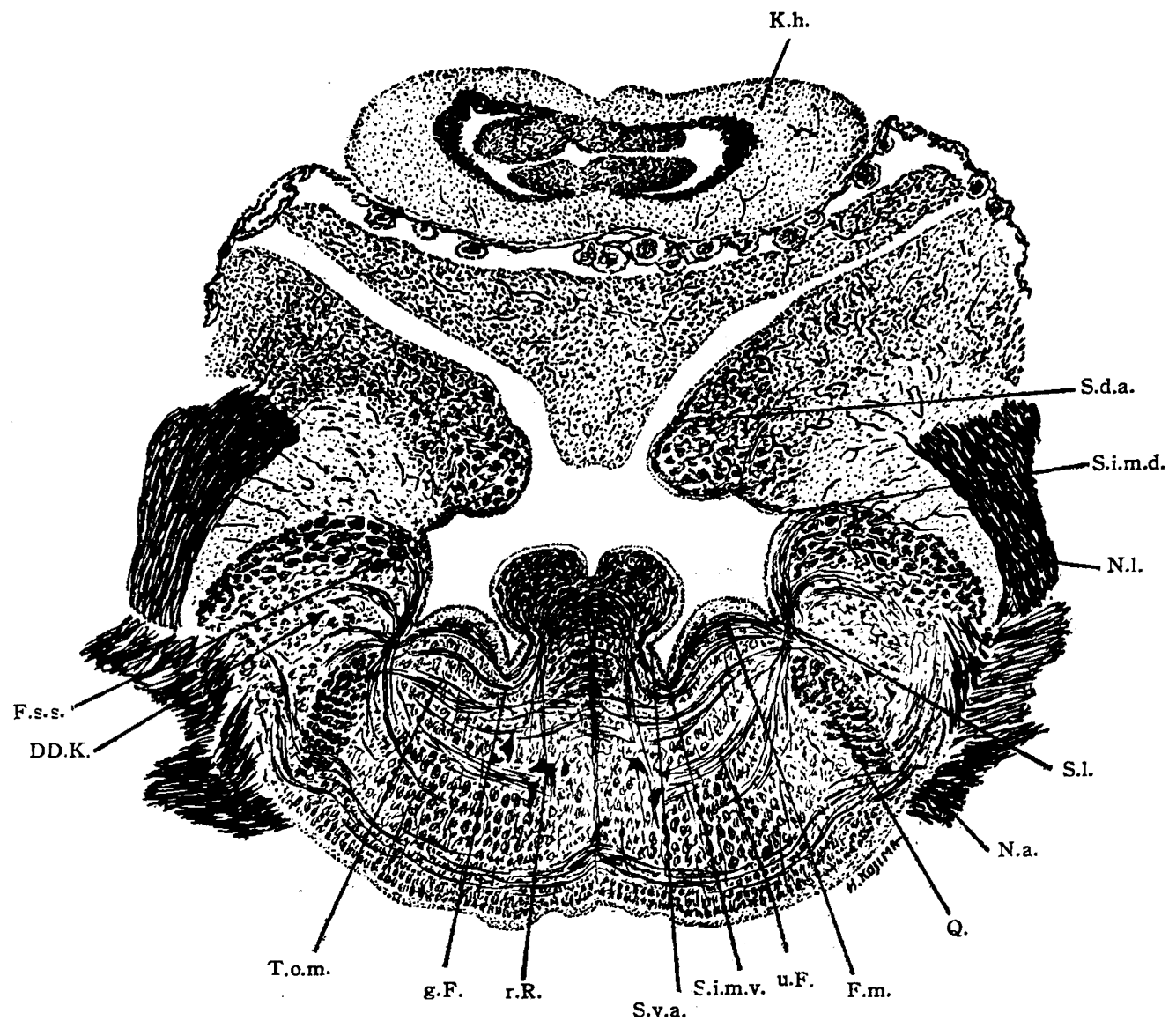

Fig. 3.

Retikuläre Zellen, Tractus reticularis und Tractus octavo-motorius an der Eminen tia anterior. D.K. Deiters scher Kern, F.m. Facialis motorische Fasern, F.s. Facialis sensibler Kern, g.F. gekreuzte Fasern des Tractus reticularis, K.h. Kleinhirn, N.a. Nervus acusticus, N.l. Nervus lateralis, Q. Quintus, r.R. rostrale Rhombencephalonuntergruppe der retikulären Zellen, S.d.a. Sulcus dorsalis accessorius, S.i.m.d. Sulcus intermedius dorsalis, S.i.m.v. Sulcus intermedius ventralis, S.v.a. Sulcus ventralis accessorius, S.1. Sulcus limitans, T.o.m. Tractus octavo-motorius, u.F. ungekreuzte Fasern des Tractus reticularis.

Kern, dessen Zellen sich wie die eigentlichen retikulären Zellen verhalten. Es entspricht dies Verhalten durchaus der engen funktionellen Verknüpfung dieses Kernes mit der als Nucleus motorius tegmenti' ${ }^{1)}$ zusammengefassten Einheit, zu der die retikulären Zellen gehören. Edinger rechnete ja auch bekanntlich den Deitersschen Kern (Fig. 2 u. 3) mit zum Nucleus motorius tegmenti (cf. Kuhlenbeck 1927, S. 146).

1) Centri tegmentali di Beccari-Castaldi der italien. Autoren (Ciabatti 1928). 


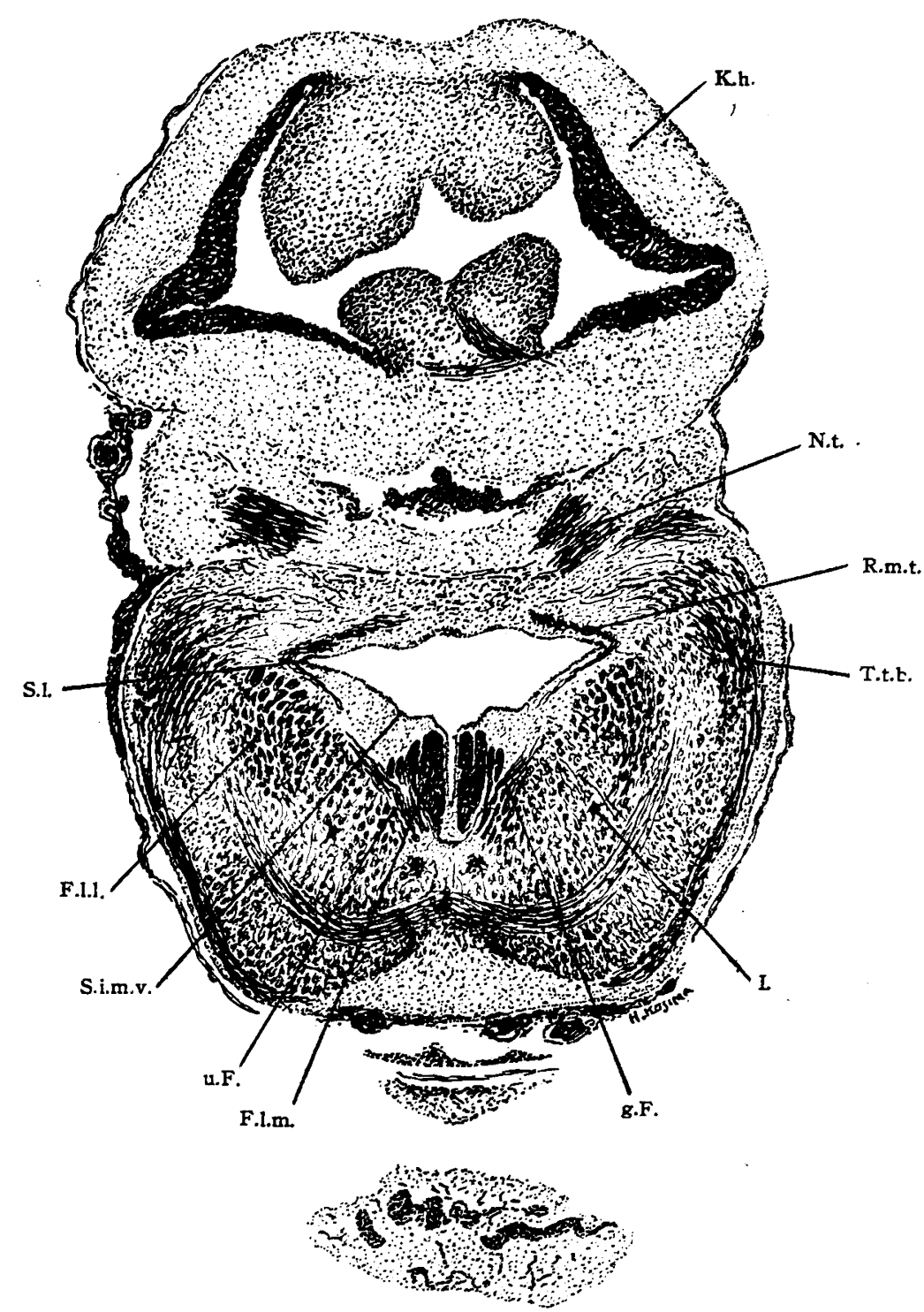

Fig. 4.

Isthmusgruppe der retikulären Zellen und Tractus reticularis an der Isthmusgegend. F.l.1. Fasciculus longitudinalis lateralis, F.l.m. Fasciculus longitudinalis medialis, g.F. gekreuzte Fasern des Tractus reticularis, I. Isthmusgruppe der retikulären Zellen, K.h. Kleinhirn, N.t. Nervus trochlearis, R.m.t. Radix mesencephalica trigemini, S.i.m.v. Sulcus intermedius ventralis, S.l. Sulcus limitans, T.t.b. Tractus tectobulbaris, u.F. ungekreuzte Fasern des Tractus reticularis. 


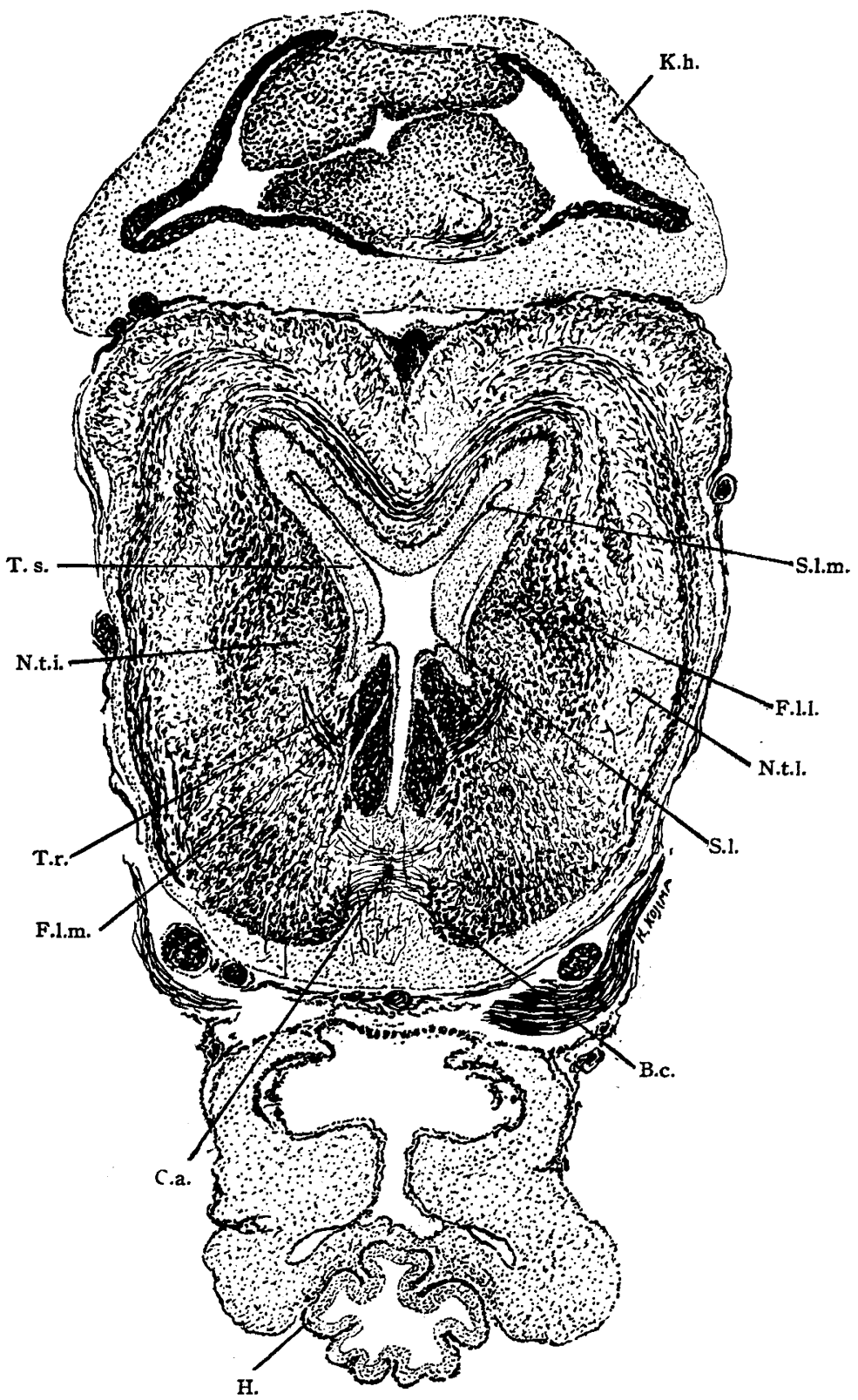

Fig. 5.

Nucleus tegmentalis internus und Tractus reticularis in der Gegend dicht kaudal vom Oculomotoriusaustritt B.c. Brachium conjunctivum, C.a. Commissura ansulata, F.1.l. Fasciculus longitudinalis lateralis, Fl.m. Fasciculus longitudinalis medialis, H. Hypophyse, K.h. Kleinhirn, N.t.i. Nucleus tegmentalis internus, N.t.l. Nucleus tegmentalis lateralis, S.l. Sulcus limitans, S.l.m. Sulcus lateralis mesen-
cephali, T.r. Tractus reticularis, T.s. Torus semicircularis. 
Aus dem oben erwähnten ergibt sich die folgende Einteilung :

Retikuläre Zellen $\left\{\begin{array}{l}\text { Mesencephalongruppe } \\ \text { Isthmusgruppe } \\ \text { Rhombencephalongruppe }\end{array}\left\{\begin{array}{l}\text { rostrale Untergruppe } \\ \text { kaudale Untergruppe }\end{array}\right.\right.$

Als verwandter Kern komint noch der Deiterssche Kern hinzu. Nucleus motorius tegmenti und Deitersschen Kern könnte man daher schliesslich auch nach dem Vorgang von Edinger als Nucleus motorius tegmenti im erweiterten Sinne bezeichnen.

3. Grösse der Zellgruppen.

Die rostralen Rhombencephalonuntergruppe ist am grössten unter allen Zellgruppen, dann kommen die kaudale Rhombencephalonuntergruppe und die Mesencephalongruppe, endlich ist die Isthmusgruppe am kleinsten.

Der Deiterssche Kern nimmt in dieser Beziehung eine Rangordnung zwischen der Mesencephalongruppe und der Isthmusgruppe ein.

4. Form und Grösse der Zellen.

Die retikulären Zellen sind polygonal, dendritenreich und viel grösser als die sonstigen Ganglienzellen des Zentralnervensystems.

5. Einteilung nach der Zellgrösse.

Die retikulären Zellen werden in zwei Arten eingeteilt : die grösseren und kleineren Zellen. Ich nenne die Ansammlung der ersteren den Nucleus reticularis major und die Ansammlung der letzteren den Nucleus reticularis minor.

Zum Nucleus reticularis major gehören die Rhombencephalongruppen d.h. die rostrale Rhombencephalonuntergruppe und die kaudale Rhombencephalonuntergruppe sowie die Mesencephalongruppe, und zum Nucleus reticularis minor die Isthmusgruppe. Auch der Deiterssche Kern verhält sich wie der Nucleus reticularis minor.

Daraus kann man sagen, dass die grösseren Zellen immer die grosseren Gruppen bilden und umgekehrt.

6. Zahl und Anordnung der Zellen.

Die Zellen sind sehr reichlich vorhanden, am reichlichsten in der 


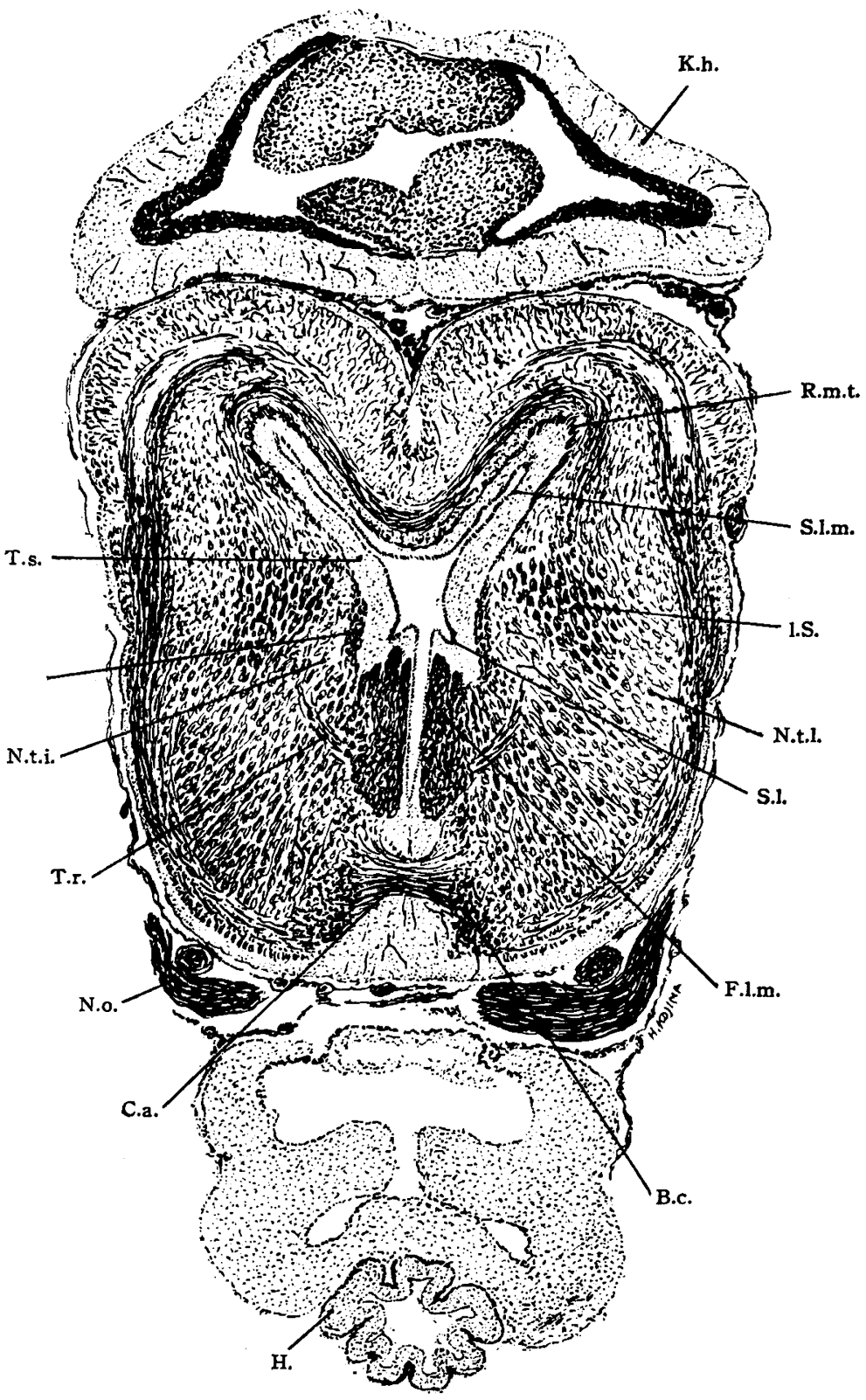

Fig. 6 .

Tractus reticularis und Nucleus tegmentalis internus an kaudalem Ende der Oculomotoriusaustrittsgegend. B.c. Brachium conjunctivum, C.a. Commissura ansulata, F.l.m. Fasciculus longitudinalis medialis, H. Hypophyse, K.h. Kleinhirn, I.S. laterale Schleife, N.o. Nervus oculomotorius, N.t.i. orales Ende des Nucleus tegmentalis internus, N.t.l. Nucleus tegmentalis lateralis, R.m.t. Radix mesencephalica trigemini, S.l. Sulcus limitans, S.l.m. Sulcus lateralis mesencephali, T.r. Tractus reticularis, T.s. Torus semicircularis. 


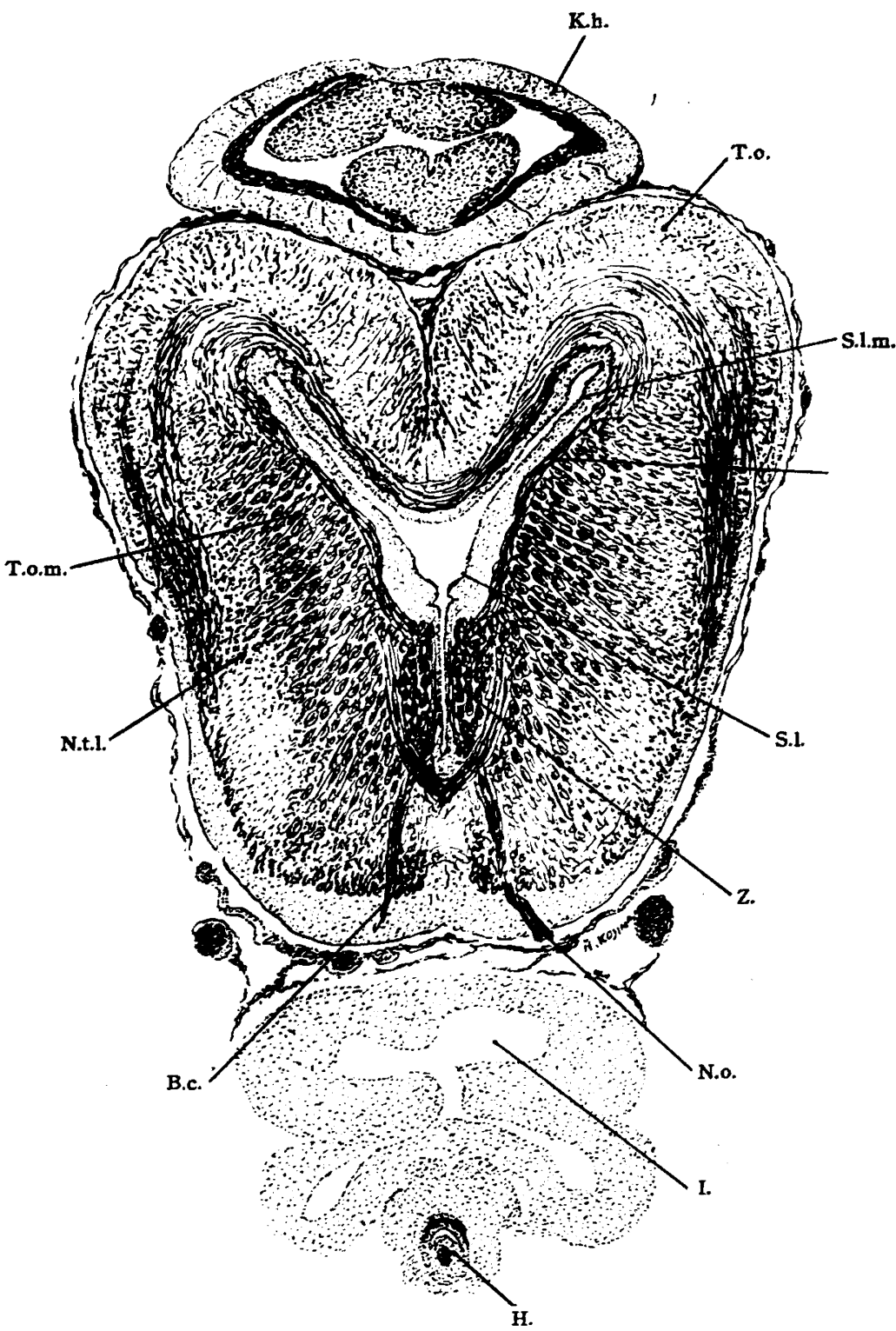

Fig. 7 .

Verhalten an der Oculomotoriusaustrittsgegend. B.c. Brachium conjunctivum, H. Hypophyse, I. Infundibulum, K.h. Kleinhirn, N.o. Nerrus oculomotorius, N.t.l. Nucleus tegmentalis lateralis, S.l. Sulcus limitans, S.l.m. Sulcus lateralis mesencephali, T.o. Tectum opticum, T.o.m. Tractus octaromesencephalicus et bulbomesencephalicus, Z. Zentrallängsbündel. 


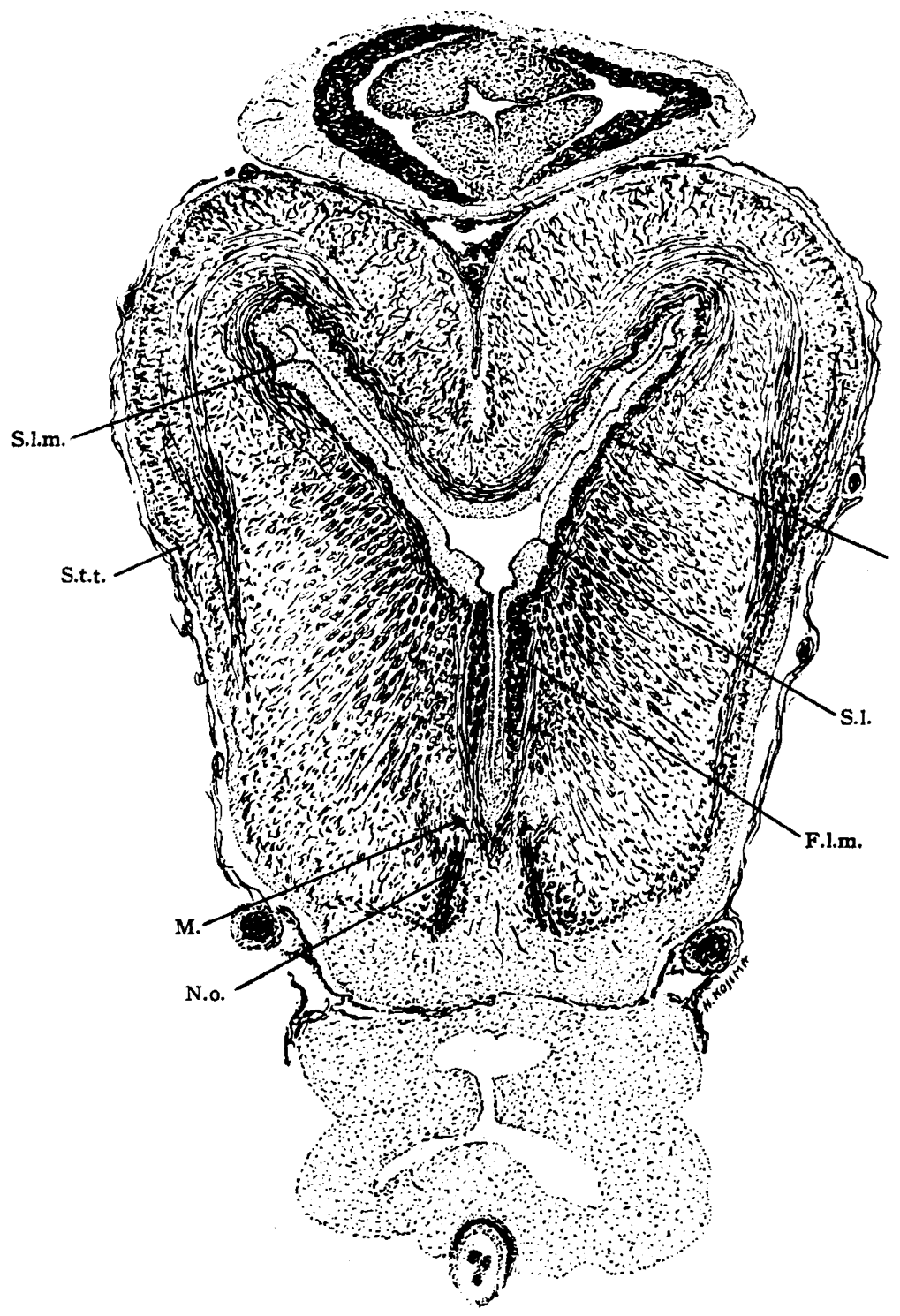

Fig. 8.

Mesencephalongruppe der retikulären Zellen dicht kaudal der Oculomotriusaustrittsgegend. F.l.m. Fasciculus longitudinalis medialis, $\mathbf{M}$. Mesencephalongruppe der retikulären Zellen, N.o. rostrale Wurzelfasern des Nervus oculomotorius, S.l. Sulcus limitans, S.l.m. Sulcus lateralis mesencephali, S.t.t. Sulcus tectotegmentalis. 
rostralen Rhombencephalonuntergruppe, damn in der kaudalen Rhombencephalonuntergruppe und der Mesencephalongruppe, und am geringsten in der Isthmusgruppe. Der Deiterssche Kern würde hier wieder zwischen Mesencephalongruppe und Isthıusgruppe einzuordnen sein.

Es scheint mir, dass die Anordnung der Zellen meist symmetrisch, paarig ist, doch hier und da ist sie nicht immer so regelmässig $\mathrm{zu}$ finden.

Die Zellen gruppieren sich so reichlich, dass sie voneinander nur wenig entfernt sind.

7. Lokalisation der Zellen.

Die Lokalisation der Zellen ist auf das Gebiet ventral vom Sulcus limitans ${ }^{\text {1) }}$ beschräukt. Der Deiterssche Kern liegt dagegen mit seinen Zellen dorsal dieses letzteren Sulcus.

Die retikulären Zellen liegen innmer dicht neben dem Fasciculus longitudinalis medialis, lateral und ventrolateral davon. Im Mesencephalon berühren sie den rostralen Teil der Oculomotoriuswurzelfasern und verbreiten sich weiter rostral bis zur Commissura transversa. Sie liegen direkt ventral und etwas lateral vom Fasciculus longitudinalis medialis, zwischen dem Sulcus intermedius ventralis ${ }^{2)}$ und dem Sulcus ventralis accessorius ${ }^{3)}$, nämlich in der Pars propria des Ventralgebietes ${ }^{4}$.

Beim rostralen Abschnitt (Fig. 4) des Rhombencephalon befinden sich die Zellen in der Isthmusgegend, sie werden daher zweckmässig als Isthmusgruppe zusammengefasst, sehr weit nach lateral und etwas ventral vom Fasciculus longitudinalis medialis entfernt, zwischen Sulcus limitans und intermedius ventralis, nämlich in Intermedioventralgebiet ${ }^{5)}$, ventromedial vom Fasciculus longitudinalis lateralis (Tractus bulbomesencephalicus).

In der eigentlichen Medulla oblongata befindet sich innerhalb des Gebietes der Acusticuskerne der Deiterssche Kern, weit dorsalwärts und lateralwärts vom Fasciculus longitudinalis medialis verschoben, dorsal des Sulcus limitans, lateral vom Intermediodorsalgebiet, zu welch letzterem nur der unmittelbar dem Ventrikel anliegende Abschnitt zu rechnen ist.

Die rostrale Rhombencephalonuntergruppe liegt in der Eminentia

1), 2), 3) u. 4). Nach meiner Untersuchung verschwindet der Sulcus limitans schon im Mensencephalon und setzt sich nicht im Diencephalon fort, wie es auch bei Entosphenus der Fall ist. 1. c.

5). Cf. Kuhlenbeck, H., Betrachtungen ïber den funktionellen Bauplan des Zentralnervensystems, Folia Anatomica Japonica. Band IV, '26; ferner Vorlesungen über das Zentralnervensystem der Wirbeltiere. Jena '26. 
anterior, in der ganzen rostralen Hälfte der eigentlichen Medulla oblongata verbreitet, lateral und ventral vom Fasciculus longitudinalis medialis, zwischen Sulcus intermedius ventralis und Sulcus accessorius ventralis, in der Pars propria ${ }^{1)}$ des Ventralgebietes. Die Zellen gruppieren sich besonders reichlich in der Octavus- und Trigeminuswurzelaustrittsgegend.

Die kaudale Rhombencephalonuntergruppe liegt in der Eminentia posterior, nämlich in der Vaguswurzelaustrittsgegend, vom Fasciculus longitudinalis medialis sehr weit nach ventromedial entfernt, zwischen Sulcus intermedius ventralis und ventralis accessorius, nach ventromedial in der Pars propria des Ventralgebietes verschoben.

Daraus sieht man, dass die rostrale und die kaudale Rhombencephalonuntergruppe und die Mesencephalongruppe am ventralsten und medialsten unter allen retikulären Zellen im ganzen Gehirn, in der Pars propria des Ventralgebietes liegen. Die Isthmusgruppe liegt viel dorsolateraler als die ersteren verschoben, im ventromedialen Teil des Intermedioventralgebietes. Der Deiterssche Kern liegt natürlich am weitesten dorsalwärts, dorsal des Sulcus limitans, in der Flügelplatte lateral vom Intermediodorsalgebiet"2.

Das folgende Schema zeigt die Lokalisation und Grösse der einzelnen Zellgruppen in horizontaler Projektion (Fig. 9).

Die die einzelnen retikulären Zellgruppen verbindende Linie zeigt eine nach lateral vorragende Kante, deren Gipfel wohl der Isthmusgruppe entspricht, welche am weitesten entfernt von der Medianlinie und am höchsten liegt.

Zieht man dagegen eine Linie, welche den mit den retikulären Zellen eng verwandten Deitersschen Kern mit einbezieht, so überschreitet diese Linie, deren Gipfel nun natürlich dem Deitersschen Kern entspricht, den Sulcus limitans. Die Mesencephalongruppe und die kaudale Rhombencephalonuntergruppe liegen am ventralsten und am nächsten der Medianlinie. In der Medianraphe des Gehirns, nämlich in der Pars accessoria des Ventralgebietes ${ }^{3)}$ kommen die Zellen nicht vor.

Im allgemeinen, in der Gegend, wo die Ganglienzellen, besonders der motorischen Kernen gut entwickelt sind, sind die retikulären Zellen auch sehr gross. Ich bin der Meinung, dass dies auf den gegenseitigen Beziehungen zwischen den beiden Zellarten beruht.

1). Saito, T., Über das Gehirn des japanishchen Flussneunauges, Entosphenus japonicus Martens, Folia Anatomica Japonica. Band VIII, Heft 3-4, 1930.

2). Cf. Kuhlenbeck, H., l. c.

3). Saito, T., l. c. 
Tamesuke Saito,

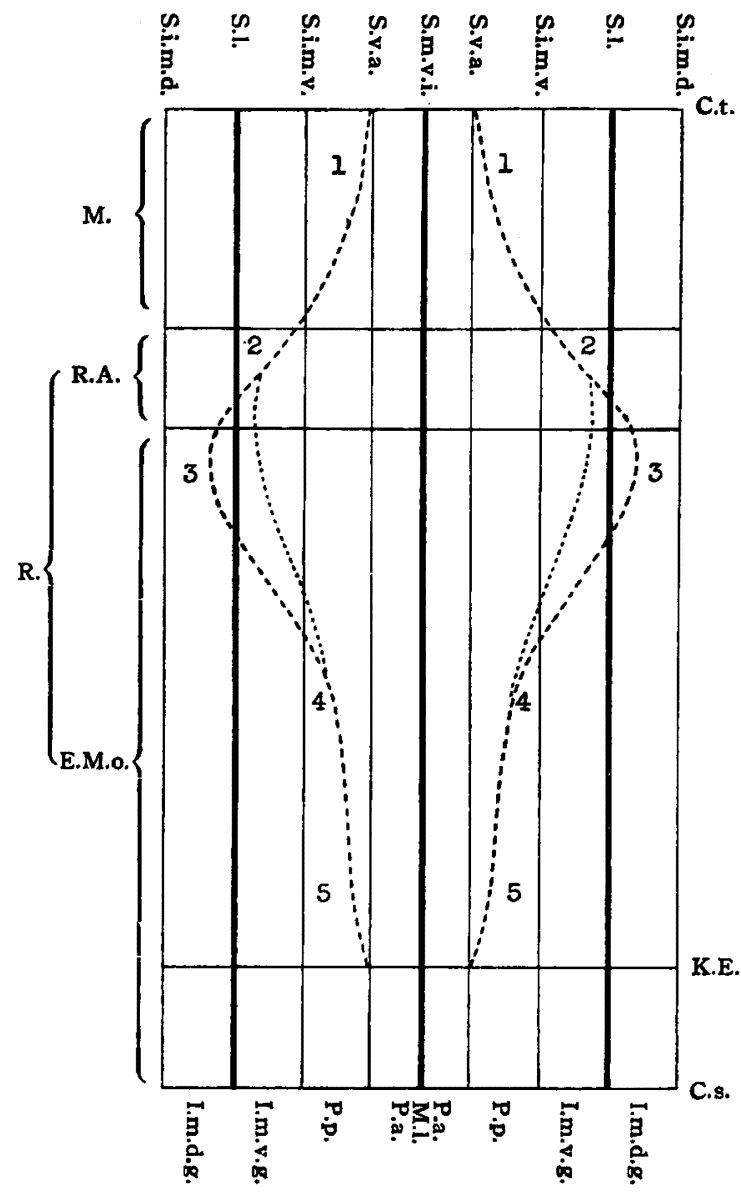

Fig. 9.

Schematische Darstellung der retikulären Zellen im Gehirn des japanischen Dornhaies (Acanthias) in horizontaler Projektion. Die gestrichelten beiderseitigen Iinien begrenzen das Gebiet des "Nucleus motorius tegmenti “ im erweiterten Sinne nach Edinger, d.h. einschliesslich des zur Flügelplatte gehörenden Deitersschen Kerns. Die punktierten I inien begrenzen das Gebiet der nur der Grundplatte angehörenden retikulären Zellen im engeren Sinne. Die Zahlen bezeichnen die Zellgruppen. 1. Mesencephalongruppe, 2. Isthmusgruppe, 3. Deitersscher Kern, 4. Rostrale Rhombencephalonuntergruppe, 5. Kaudale Rhombencephalonuntergruppe, C.t. Commissura transversa, C.s. Calamus scriptorius, E.M.o. Eigentliche Medulla oblongata, I.m.d.g. Intermediodorsalgebiet, I.m.v.g. Intermedioventralgebiet, K.E. Kaudales Ende der Eminentia posterior, M. Mesencephalon, M.l. Medianlinie, P.a. Pars accessoria des Ventralgebietes, P.p. Pars propria des Ventralgebietes, R. Rhombencephalon, R.A. Rostraler Abschnitt des Rhombencephalon, S.i.m.d. Sulcus intermedius dorsalis, S.i.m.v. Sulcus intermedius ventralis, S.l. Sulcus limitans, S.m.v.i. Sulcus medianus ventralis internus, S.v.a. Sulcus ventralis accessorius. 
8. Fasersysteme.

Es ist bekannt, dass die Fasern der retikulären Zellen Gehirn und Rückenmark miteinander verbinden und Reize kaudalwärts korrelieren.

Sie sind sehr gut entwickelt und werden in aufsteigende and absteigende eingeteilt. Ihr grösster Teil ist gekreuzt, während ein geringer, besonders kleiner Teil aus den lateralen Zellen der rostralen Rhombencephalonuntergruppe ungekreuzt bleibt.

\section{a). Gekreuzte Fasern.}

Die gekreuzten Fasern in der eigentlichen Medulla oblongata (Fig. 1-3) steigen fast vertikal nach dorsal auf, treten in den Fasciculus longitudinalis medialis von lateral ein und kreuzen in der Medianraphe des letzteren; sie steigen auch im gegenseitigen Intermedioventralgebiet auf, entlang der Ventrikelwand und kommen direkt dorsal vom Sulcus intermedius ventralis. Sie werden rostraler vorschreitend, desto stärker; so sind sie in der Eminentia posterior sebr stark, rostral von der Eminentia anterior arn stärksten im ganzen Gehirn. Sie steigen mit den motorischen Facialiswurzelfasern an der Ventrikelwand, unter dem Ependym nach dorsal vorn auf und liegen immer ventral vom Sulcus limitans.

Im rostralen Abschnitt (Fig. 4) des Rhombencephalon ist der Faserverlauf gauz gleich wie bei der eigentlichen Medulla oblongata; sie sind nur etwas schwächer als dort.

In der kaudalen Hälfte (Fig. 5) des Mesencephalon sind die retikulären Fasern sehr schwach, treten in den Fasciculus longitudinalis medialis ein und kreuzen damit unter dem letzteren, direkt dorsal vom Brachium conjunctivum.

In der rostralen Hälfte (Fig. 6-8) des Mesencephalon sind die Fasern stark und kreuzen mit den anderseitigen immer dorsal der Commissura ansulata.

Nach ihrer Kreuzung steigen sie nach dorsal auf, entlang der gegenseitigen Veutrikelwand, um endlich in der Ganglienzellengruppe zu endigen, die ventral von Torus semicircularis (Fig. $5 \mathrm{u.6}$ ), medioventral von der lateralen Schleife des Mesencephalon liegt. Diese Stelle liegt kaudoventral von der Radix mesencephalica trigemini, zwischen dem Sulcus intermedius ventralis und Sulcus limitans, im Intermedioventralgebiet.

Da die betreffenden Ganglienzellen ventral vorn Torus semicircularis sich befinden, so lassen die beiden sich voneinander klar unterscheiden. 
Ich nemne diesen Kern den Nucleus tegmentalis internus. Er ist von der lateralen Schleife scharf begrenzt, viel kleiner als der Nucleus tegmentalis lateralis (Fig. 5 u. 6).

Das Faserbündel der retikulären Zellen ist sehr lang, liegt zwischen dem Nucleus tegmentalis internus und dem Rückenmark, immer ventral vom Sulcus limitans. Ich nenne dieses Bündel den Tractus reticularis.

Die von der Wurzelgegend der Nervus octavus und Nervus lateralis anterior (Fig. 2 u. 3), nämlich vom Deitersschen Kern entstammenden Fasern steigen ventral vom Tractus reticularis in sehr breiten Zügen ab, parallel zur Ventrikelwand, kreuzen mit den anderseitigen in der ventrai von Fasciculus longitudinalis medialis liegenden Raphe; dann steigen sie wieder parallel zu den Fassern dieses Tractus auf, gehen durch den rostralen Abschnitt des Rhombencephalon, treten im Mesencephalon ein und vereinigen sich endlich mit dem Tractus reticularis.

Dieses Bündel dürfte dem von Kappers u. a. beschriebenen Tractus octavo-motorius entsprechen.

Die Unterscheidung zwischen dem Tractus reticularis und dem Tractus octavomotorius ist im Rhombencephalon durchaus klar, aber im Mesencephalon unklar. Hier vereinigen sich die beiden miteinander und bilden ein sehr schwaches Faserbündel. Der Faserschenkel vor ihrer Kreuzung ist in der Eminentia posterior der eigentlichen Medulla oblongata schwach, nach medial etwas konvex, in der Eminentia anterior am stärksten im ganzen Gehirn, nach medial konkav und im rostralen Abschnitt (Fig. 4) des Rhombencephalon etwas schwach und sein ventrales Ende klafft nach lateral; und endlich im Mesencephalon (Fig. 5-8) ist er sehr schwach und verlaüft fast senkrecht, Er liegt immer entlang der lateralen Seite des Fasciculus longitudinalis medialis.

Der gekreuzte Teil des Tractus reticularis bildet an der Ventralraphe des Ventrikels eine vertikale Kreuzungslinie, die ich die Decussatio longitudinalis mediana (Fig. 1-3) nenne, und ist in der ganzen Länge des Rhombencephalon hindurch zu finden.

b). Ungekreuzte Faseru.

Die ungekreuzten Fasen (Fig. 1-4) der retikulären Zellen zeigen ventral vom Sulcus intermedius ventralis eine mediale Konvexität und steigen mit ihren gekreuzten Fasern ventral vom gleichseitigen Ependym auf.

Sie stammen aus allen Gruppen der retikulären Zellen, jedoch nicht aus dem Deitersschen Kern, die grössten kommen aus dem lateralen Teil der rostralen Rhombencephalonuntergruppe. 
Manche Fasern des Tractus reticularis gelangen vom rostralen Teil des Mesencephalon kaudalwärts his zum Rückenmark.

\section{Vergleichung und Zusammenstellung.}

1). Retikuläre Zellen.

Kappers ('20) fand die retikulären Zellen der Plagiostomen ,, auf gewissen Distanzen roneinander getremnt." Nach meinem Befund scheint es auch beim Squalus, dass die Zellgruppen auch durch gewisse Distanzen voneinander getremnt sind, während die nicht so grosse Entfernung zwischen den einzelnen Zellen sich nicht sicher messen lässt.

Der ron ran Hoevell ('11) bei Rochen und von Kappers ('20) bei Plagiostomen genamnte Nucleus reticularis inferior entspricht selbstverständlich meiner kaudalen Rhombencephalonuntergruppe.' Die beiden Autoren sagen wie folgt : Die retikulären Elemente des Vagusareales zeigen sich jedoch sehr zahlreich und sind ventral in der Raphe angehäuft. Sie können dort so zahlreich sein, dass man von einem wirklichen Kern : dem Nucleus reticularis inferior, reden kamn (v. Hoevell), der sich nicht nur in der Raphe, sondern auch seitlich davon guirlandenförmig unter dem Fasciculus longitudinalis centralis ausdehnt.

Nach meiner Untersuchung liegen die retikulären Zellen grösstenteils nicht in der ventralen Raphe, nämlich in der Pars accessoria des Ventralgebietes ${ }^{1}$, sondern seitlich davon wohl in der Pars propria des Ventralgebietes ${ }^{2}$.

Der Nucleus reticularis medius nach ihmen entspricht hauptsächlich meinem Deitersschen Kern und der kaudalen Hälfte der rostralen Rhombencephalonuntergruppe und ihr Nucleus reticularis superior wahrscheinlich meiner rostraler Hälfte der rostralen Rhombencephalonuntergruppe und der Isthmusgruppe. Ich sah eine klare Grenze zwischen der rostralen Rhombencephalonuntergruppe und der Isthmusgruppe, die beiden gehen nicht ineinander über.

Die betreffenden Autoren sagen auch: ,, Die Oculomotoriusgruppe weist nur sehr wenig Zellen in der Raphe auf.-und bleiben ziemlich dorsal nahe dem lateralen Rande des Fasciculus longitudinalis centralis, wo auf jeder Seite 6-8 solcher Zellen in einem Schnitte gefunden werden kömmen."

Elemente, welche den retikulären Zellen entsprechen, fand ich im Deitersschen Kern, welcher der Oktavusgruppe von beiden Autoren

1) ט. 2). 1. c. 
vielleicht teilweise entspricht, nicht so reichlich, höchstens nur 6 an der Zahl in einem Frontalschnitt.

Kappers ('20) äussert sich bei Plagiostomen : ,, Einige Zellen bilden jedoch eine mehr ventrolaterale Gruppe, welche offenbar mit eintretenden Vestibularisfasern in Verbindung steht,- ."

Ich meine, dass dieser Kern wohl meinem Deitersschen Kern entspricht, der im ventromedialen Teil der Flügelplatte, unmittelbar lateral des Intermediodorsalgebiets liegt.

Die beiden Autoren schreiben noch: ,, Auch die Zellen dieses retikulären Kernes (Nucleus reticularis superior) liegen in derselben Weise geordnet, seitlich von der Raphe (nicht in der Raphe). Sie beschränken sich hauptsächlich auf das obere Drittel des Querschnittes.“

Die ihrem Nucleus reticularis superior entsprechende rostrale Hälfte der rostralen Rhombencephalonuntergruppe sowie die Isthmusgruppe liegen im Intermedioventralgebiet, also nicht in der Raphe. Ihr Nucleus reticularis mesencephalicus entspricht wohl meiner Mesencephalongruppe und liegt in der Pars propria des Ventralgebietes.

\section{2). Über den Faserverlauf.}

Nach der von Kappers ('06 u. '21) bei Selachiern und Plagiostomen angegebenen Beschreibung entspricht der Tractus cerebellomotorius cruciatus et rectus wahrscheinlich teilweise meinem Tractus reticularis. Er sagt über diese Bahn das folgende : ,, Diese Bahn entsteht wahrscheinlich hauptsächlich aus den Aurikeln des Kleinhirns und - weil dort keine Kerne vorkommen und die Axonen der Körnerzellen einen anderen Verlauf nehmen - vermutlich aus Zellen, welche den Purkinjeschen Zellen verwandt sind.“

Doch wenn man das Präparat mit der eingangs angegebeuen Färbung verfolfgt, so zeigt der Tractus reticularis die Dunkelblaufarbe und dagegen sind die von Kappers genannten Fasern, die aus dem Auriculus cerebelli kommen, schwarzbraun; die Differenzierung zwischen den beiden Fasern ist ausserordentlich klar.

Diese Fasern gehen im Nucleus tegmentalis internus des Mesencephalon verloren und hängen nicht mit dem Auriculus cerebelli zusammen. Es ist mir wahrscheinlich, dass die Fasern, die nach Kappers mit dem Auriculus cerebelli zusammenhängen, andere als die meines Tractus reticularis sind. Doch scheint mir der Tractus cerebellomotorius von Kappers meinen Tractus reticularis und noch die aus dem Auriculus cerebelli kommenden Fasern zu umfassen. Nach der Palschen Original- 
Markscheidenfärbungsmethode ist diese Differenzierung unmöglich wegen der gleichmässigen dunkelblauen Färbung aller Fasern.

Anderseits ist der Verlauf meines Tractus reticularis von dem des Tractus cerebellomotorius cruciatus et rectus ganz verschieden. Nämlich der Tractus reticularis d.h. die mit dem Nucleus tegmentalis internus zusammenhängenden Fasern verlaufen entlang der Ventrikelwand des Mesencephalon, treten direkt ventral vom Sulcus ventralis accessorius in das Zentrallängsbündel des Rhombencephalon ein und kreuzen in der Medianraphe mit den gegenseitigen.

Über den Tractus octavomotorius sagt Kappers ('20): ,, Die Endkerne des $\mathrm{N}$. lateralis und Vestibularis sind kommissurell verbunden mittels Fasern, die im ventralen Tegmentum kreuzen. Aus der ganzen Area statica entstehen ausserdem reflektorische und koordinatorische Fasern, welche auf- und absteigend im hintern Längsbündel und darunter verlaufen und den Tr. octavo-motorius darstellen. Sie verlaufen mit den zerebello-motorischen Fasern nahe an dem Ventrikel entlang als dorsale Bogenfasern zum prädórsalen Bündel, gekreuzt und ungekreuzt. Die grosse Mehrheit dieser Fasern hat einen aboralen Verlauf. Nur wenige steigen zu den vorderen Augenmuskelkernen auf. Die grossen Zellen, denen sie entstammen, liegen in dem dorsolateralen Rand der Oblongata und sind vielleicht teilweise dem Deiters-Kern zu vergleichen, obwohl sie noch nicht so kompakt zusammen liegen."

Daraus kann man entnehmen, dass mein Tractus octavomotorius nicht vollständig mit dem gleichnamigen Faserzug von Kappers übereinstimmt, sondern nur demjenigen Anteil des Kappersschen Tractus entspricht, der aus dem Deitersschen Kern entstammt. Dieser Auteil ist meiner Ansicht nach nur gekreuzt.

\section{Schluss.}

1). Wenn man $40-50 \mu$ dicke Schnitte mit $0.5-1.0 \% \quad(0.5 \%)$ Kalipermanganat-Lösung statt $0.25 \%$ nach der $\mathrm{Palschen}$ Originalmethode 5-10 Minuten lang färbt und auf 1-2 Stunden differenziert, so werden die retikulären Zellen dunkelbraun gefärbt und die Markscheide schwarzblau, wodurch man die beiden leicht ganz klar unterscheiden kann.

2). Die retikulären Zellen kommen im grössten Teil des Rhombencephalon nämlich in der eigentlichen Medulla oblongata, im rostralen Abschnitt des Rhombencephalon sowie im Mesencephalon vor, sie fehlen im Übergangsgebiet des Rhombencephalon in das Rückenmark, im Diencephalon und im Telencephalon. Die vorderste Zelle liegt in der 
Gegend der Commissura transversa des Mesencephalon, die kaudalste in der Gegend des kaudalen Endes der Eminentia posterior der eigentlichen Medulla oblongata.

3). Die retikulären Zellen können in drei Gruppen, die Mesencephalongruppe, die Isthmusgruppe und die Rhombencephalongruppe eingeteilt werden. Die Rhombencephalongruppe teilt sich wieder in zwei Untergruppen ein, die rostrale und die kaudale Untergruppe.

4). Eng verwandt mit den der Grundplatte angehörigen retikulären Zellen, jedoch gesondert einzuteilen sind die Elemente des Deitersschen Kerns, welche dorsal des Sulcus limitans in der Flügelplatte, lateral vom Intermediodorsalgebiet liegen. Dieser Kern befindet sich im Octavuseintrittsgebiet, dorsolateral vou der rostralen Rhombencephalonuntergruppe.

5). Am grössten ist die rostrale Rhombencephalonuntergruppe, dann folgen die kaudale Rhombencephalonuntergruppe, die Mesencephalongruppe und endlich ist am kleinsten die Isthmusgruppe. Der Deiterssche Kern rangiert zwischen Mesencephalon- und Isthmusgruppe.

6). Die Form der retikulären Zellen ist polygonal ; sie sind dendritenreich und bedeutend grösser als die sonstigen Ganglienzellen des Zentralnervensystems.

7). Nach der Grösse kamn man die retikulären Zellen in zwei Arten, in den Nucleus reticularis major und den Nucleus reticularis minor einteilen. Im allgemeinen sind die Zellen desto grösser, je grösser die Gruppen sind.

8). Die retikulären Zellen kommen immer in der Pars propria des Ventralgebietes und im Intermedioventralgebiet vor. Sie liegen immer entlang dem Fasciculus longitudinalis medialis (Zentrallängsbündel). Die eng mit den retikulären Zellen verwandten Elemente des Deitersschen Kerns liegen dorsal des Sulcus limitans, in der Flügelplatte, lateral vom Intermediodorsalgebiet.

9). Ich nenne die Fasern der retikulären Zellen den Tractus reticularis. Der Tractus reticularis enthält aufsteigende und absteigende Fasern. Er ist zum grössten Teil gekreuzt, teils ungekreuzt. Mit dem Tractus reticularis ist eng verbunden der Tractus octavomotorius aus dem Deitersschen Kern.

10). Das rostrale Ende des Tractus reticularis stellt der Nucleus tegmentalis internus des Mesencephalon dar.

11). Der Tractus reticularis bildet durch die ganze Länge des Rhombencephalon die Decussatio longitudinalis mediana.

Herr Dozent Dr. H. Kuhlenbeck, vorher an der Keio-, jetzt an 
der Universität Breslau und Herr Professor Dr. K. Okajima haben mir in hohem Masse ihre freundliche Leitung und anregende Unterstützung bei dieser Arbeit gegebeu.

Zum Schluss ist es mir eine angenehrne Pflicht, den obigen beiden Herren Professoren hierfür meinen herzlichsten Dank auszusprechen.

\section{Literaturverzeichnis.}

1). Ciabatti, O., Contributi alla conoscenza del Cyprinodon (I.ebias) calaritanus. I centri tegmentali die Beccari-Castaldi. Scritti Biolog. Vol. IV, 1928.

2). Hoevell, van, Remarks on the reticular cells of the oblongata in different vertebrates, Proceed. of the Kon. Akad. V. Wetensch, Amsterdam April 1911.

3). Kappers, Ar., The structure of the Teleostean and Selachian brain, Journ of Comp. Neurolog. Vol. 16, 1906.

4). —_ - Die vergleichende Anatomie des Nervensystems der Wirbeltiere und des Menschen, I und II Abschnitt, 1920 und 1921.

5). Kuhlenbeck, H., Betrachtungen über den funktionellen Bauplan des Zentralnervensystems, Fol. Anat. Jap. Bd. IV, Hef. 2, 1926.

6). - - Vorlesungen über das Zentralnervensystem der Wirbeltiere, Jena 1927.

7). Saito, T., Ưber das Gehirn des japanischen Flussneunauges, Entosphenus japonicus Martens, Fol. Anat. Jap. Bd. VIII, Hef. 3-4, 1930.

8). Sterzi, G., Il sistema nervoso centrale nei Vertebrati. Vol. II, Pesci. Padova 1909-1912.

9). Stieda, Untersuchungen über das centrale Nervensystem der Knochenfische, Zeitschr. f. Wissenschaftl. Zoolog. 18, 1868. 